\title{
The role of defects in the crystal/quasicrystal transformation
}

\author{
P DONNADIEU*, A PROULT and C RICOLLEAU ${ }^{\dagger}$ \\ Laboratoire d'Etudes des Microstructures, CNRS/ONERA, BP 72, 92322 Chatillon, \\ Cedex, France \\ ${ }^{\dagger}$ Laboratoire de Minéralogie-Cristallographie, Universités Paris VI-VII, 4 place Jussieu, \\ 75252 Paris, Cedex 05, France
}

\begin{abstract}
The transformation between quasicrystals and related crystals, the so-called approximant phases, appears as a major point in the understanding of quasicrystal stability. Structural defects of approximant phases seem to be involved in the mechanism of crystal/quasicrystal transformation. Theoretical works as well as observations are supporting this point of view. We here report observations made by transmission electron microscopy on two systems which provide relevant examples for two types of mechanisms. In both cases, the approximant defects are identified as antiphase boundaries. In the first system $(\mathrm{Al}-\mathrm{Li}-\mathrm{Cu})$, the transformation is due to a progressive organization of the approximant phase defects. In the second system ( $\mathrm{Fe}-\mathrm{Cr}-\mathrm{Mo}$ ), the vertex of intersecting defects exhibits key structural feature for the transformation in a quasicrystal.
\end{abstract}

Keywords. Quasicrystal; phase transformation; defect; antiphase boundary.

\section{Introduction}

Since their discovery, quasicrystals keep being an astonishing state of matter. The increasing quality of the samples has definitely ruled out the possibility of a non-crystallographic symmetry resulting from twinning. First, quasicrystals were described as icosahedral glass, but quickly the hyperspace crystallography appears as the best tool to account for these unusual structures. As the icosahedral quasicrystal corresponds to a 6-d lattice, its cell decoration is represented by atomic surfaces which are still matter of investigation. The hyperspace description has been strengthened by the observation of microstructural features which can be described as equivalent of the classical crystal defects (dislocations and antiphase boundaries). However, it is out of the scope of this paper to give details about the general properties of quasicrystals (for an introduction and a survey of quasicrystal properties, see Janot (1992) and Hippert and Gratias (1994)). Though we have an efficient and consistent description of the quasicrystal structure and microstructure, one of the more important question is still a matter of debate: that is the stability of quasicrystal. It remains unclear whether the quasicrystal stability derives from energy or entropy. Therefore, diffuse scattering in quasicrystal becomes of increasing interest. Detailed studies are also devoted to the phase diagram because they reveal the existence of numerous crystalline phases in the vicinity of the quasicrystal state. These phases called approximant are crystalline and exhibit icosahedral local environments. According to their composition and local features, quasicrystals and their approximant counterparts are very close. They essentially differ by the existence or not of periodicity. The breaking of the translation symmetry is then a key for the approximant/quasicrystal transformation. Of course any information

\footnotetext{
*Author for correspondence
} 
on this transformation is expected to provide information about the quasicrystal stability.

Basically, a breaking of translation symmetry can be generated by defect networks. Several experimental and theoretical works have pointed out the role defects may have in the crystal/quasicrystal transition: for instance, the precursor state of the rhombohedral approximant of the $\mathrm{Al}-\mathrm{Cu}-\mathrm{Fe}$ quasicrystal (Audier et al 1991) and a quasicrystal/crystal transformation mediated by phason walls (Mosseri 1993). We have focussed on the structural defects of approximant, and, in general, icosahedral motif crystals (namely Frank-Kasper (1958) phases). The defects of interest here are planar defects which are very frequent in these phases. We have studied two systems that deserve attention because of their exemplar behaviour regarding crystal/quasicrystal transition. One case displays a progressive ordering of the defect network which turns to an aperiodic organization and thus achieves to transform the crystal in a quasicrystal. The second example shows no organized defect network but the core of defects, precisely the vertex of intersecting defects, displays key features strongly recalling a theoretical approach of the crystal/quasicrystal transformation (Kulkarni et al 1993).

\section{Transformation mediated by defect organization}

The $\mathrm{Al}_{5} \mathrm{Li}_{3} \mathrm{Cu}$ phase $(a=1 \cdot 39 \mathrm{~nm}, \mathrm{bcc}, \operatorname{Im} 3 \mathrm{~m}, 160$ atoms/cell) is an approximant of the $\mathrm{Al}_{6} \mathrm{Li}_{3} \mathrm{Cu}$ quasicrystal (Sainfort and Dubost 1986). A systematic investigation by transmission electron microscopy (TEM) has been carried out in as-cast $\mathrm{Al}_{5} \mathrm{Li}_{3} \mathrm{Cu}$ samples. The microstructure exhibits no dislocation but a high density of planar defects which intersect each other forming a 3-dimensional network. These defects are easily identified using dark field images in 2-beam condition. The fringe contrast on DF images is symmetric and the $\mathrm{Al}_{5} \mathrm{Li}_{3} \mathrm{Cu}$ phase is centrosymmetric; consequently, the planar defects are antiphase boundaries. Since symmetric contrasts on DF images are obtained for any diffracting condition, we derived that the fault vector is a simple one in a bcc structure: $1 / 4\langle 111\rangle$ (Donnadieu 1992).

In some area of the approximant phase samples, we observed very high density of defects generating extra-spots on the electron diffraction patterns (EDPs). These extra-spots are indexable with rational value which indicates that the network of defect is periodically organized. The most remarkable diffraction patterns are observed along the $\langle 023\rangle$ zone axis. This zone axis which has already some similarity with the 5-fold quasicrystal axis becomes, because of the extra-spots, very close to the 5-fold diffraction pattern (Donnadieu 1991). The indexation of the extra-spots gives the exact direction of the antiphase planes. The APB planes belong to following families: $\{1,2,3\},\{2,3,5\}$, $\{0,2,3\},\{2,3,3\}$. Amazingly, most of these planes are related to the icosahedral shape: the APB planes are close to the triacontahedron facets $(=2$-fold icosahedron axes) (Donnadieu 1992).

These preliminary observations suggest a mechanism of transformation by piling up defects. We never observe the transformation in as-cast $\mathrm{Al}_{5} \mathrm{Li}_{3} \mathrm{Cu}$ crystal samples. An evident reason is that the approximant and quasicrystal compositions are not close enough that composition fluctuations in the as-cast samples allow to vary from $\mathrm{Al}_{5} \mathrm{Li}_{3} \mathrm{Cu}$ to $\mathrm{Al}_{6} \mathrm{Li}_{3} \mathrm{Cu}$. We had to elaborate a dedicated heat treatment to get samples in which the quasicrystal coexists with its approximant phase (Degand et al 1993). 


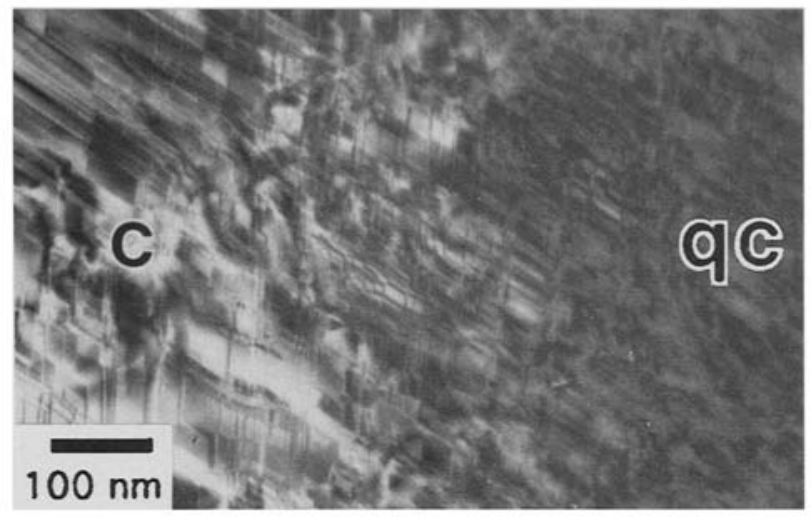

Figure 1. Interfacial area between the approximant phase and the quasicrystalline phase (respectively labelled $c$ and $q c$ ).
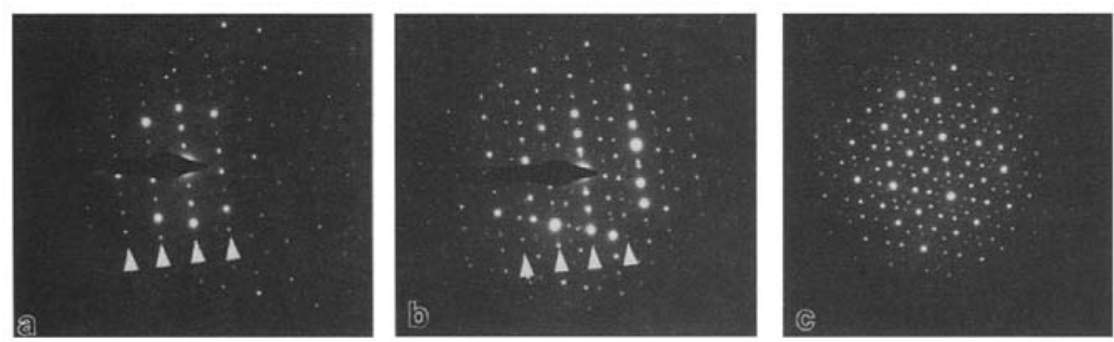

Figure 2. Transformation of the approxımant $\langle 023\rangle$ axis (a) in an icosahedral phase 5-fold axis (c). Note the aperiodic extra-spots that appear in $\mathbf{b}$ along the diffraction rows indicated by arrows.

TEM investigation of the crystal/quasicrystal interface shows first that the interface is not sharp but there is an interfacial area in which the transformation occurs. Across the interfacial area, the defect density increases from the $\mathrm{Al}_{5} \mathrm{Li}_{3} \mathrm{Cu}$ crystal to $\mathrm{Al}_{6} \mathrm{Li}_{3} \mathrm{Cu}$ quasicrystal (figure 1).

Electron diffraction patterns (EDPs) have been taken in successive areas from the crystalline phase to the quasicrystalline one (selected area: $0 \cdot 2 \mu \mathrm{m}$ ). The transformation is illustrated by the sequences of EDPs shown in figures 2 and 3. The $\langle 023\rangle$ and $\langle 111\rangle$ cubic phase directions respectively transform in 5-fold and 3-fold icosahedral axes. Both sequences of EDPs are interpretable on the basis of the organization of defects mentioned previously. However, EDPs are complex and a detailed analysis cannot be given here (for a full explanation see Donnadieu and Degand 1993). In both sequences (figures 2 and 3), the extra-spots are due to the high density of defects whose directions are close to 2-fold icosahedral axes. The $\langle 023\rangle$ EDP sequence starts with a pseudo-5 fold symmetry pattern similar to the ones observed in very faulted as-cast approximant samples. When the density of defect increases, aperiodic spots appear along the [001] reciprocal row (figure $2 \mathrm{~b}$ ) achieving the transformation into a 5-fold axis (figure 2c). The loss of intensity of the cubic lattice diffraction spots (figure $3 \mathrm{c}$ ) shows that the crystal lattice is upset by the APB organization. Finally, a selection of some diffraction spots occurs which transform the highly faulted approximant (figure $3 \mathrm{~d}$ ) in 

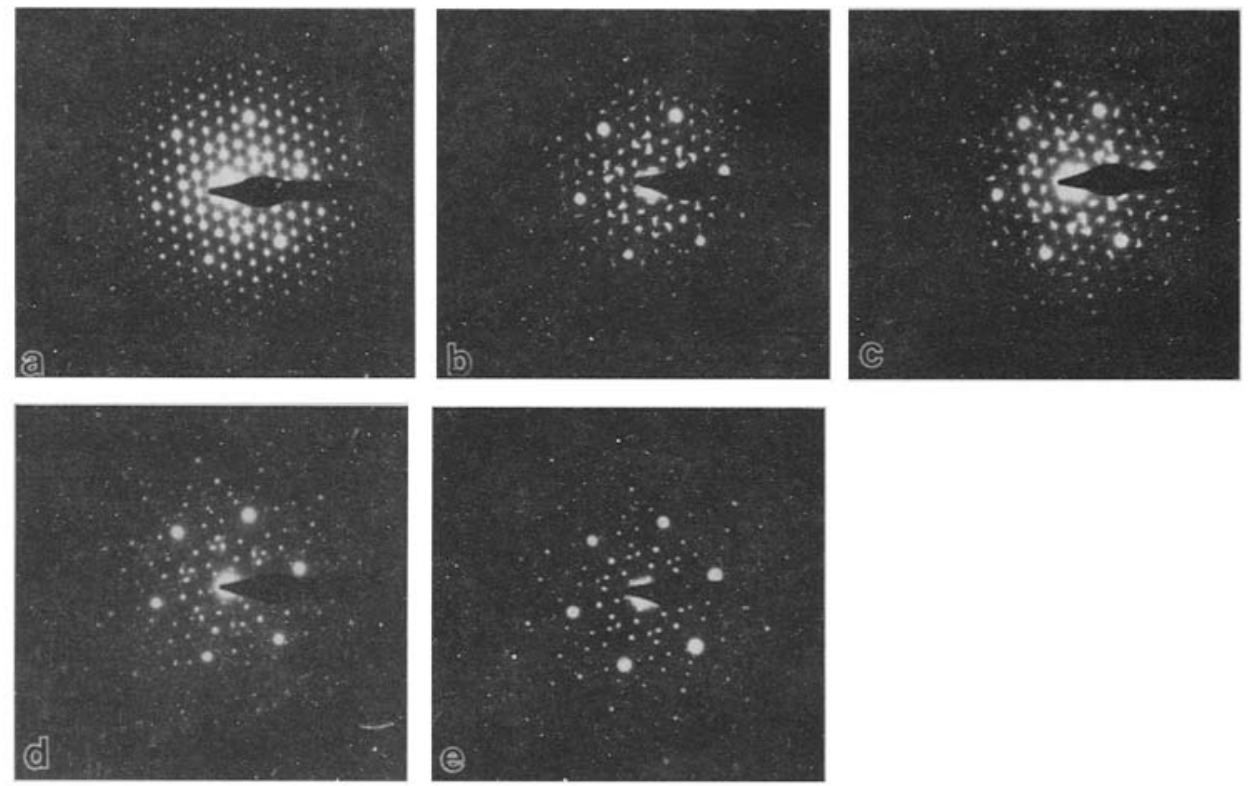

Figure 3. Sequence of EDPs along the approximant phase $\langle 111\rangle$ zone (a) axis that transforms in a icosahedral 3-fold axis (d). Note the extra-spot in b and the decrease of intensity of the approsimant phase spots in $\mathbf{c}$. The final EDP is quasicrystalline owing to an anomalous selection among the extra-spots in $\mathbf{d}$.

a quasicrystal (figure 3e). This spot selection cannot be explained in the frame of a periodic APB organization: it is the signature of an aperiodic order. This anomalous spot selection is concomitant to the aperiodic spot on the 5-fold EDP (figure 2b).

Both sequences of EDPs confirm a mechanism involving the organization of high density APB network. The defect organization which is, at once, crystalline upsets the cubic crystal lattice and finally becomes non-crystallographic. The present observations suggest that the crystal/quasicrystal transformation can be continuous. Consequently, the transformation may sometimes be incomplete and lead to reminiscence of the cubic phase. The deviations of the $\mathrm{Al}_{6} \mathrm{Li}_{3} \mathrm{Cu}$ quasicrystal to perfect icosahedral symmetry are consistent with such effect (Donnadieu 1994).

According to the TEM images, the APBs stay in well defined planes across the transformation area. Because of the composition differences the APBs are necessarily non-conservative. The increasing APB density is equivalent to a decreasing distance between APBs. The fact that an aperiodic organization appears in highly faulted area means that there is a cricital density, beyond which the APB organization is incompatible with a periodical one. Such behaviour reminds of geometrical frustration. A critical situation must be reached when the distance between defects is of the same order as the size of strong cohesion icosahedral aggregate.

The present example illustrates a role of defects which seems to have some general significance. Similar behaviour has been observed in the transformation of the hexagonal $\varepsilon \mathrm{Al}_{4} \mathrm{Cr}$ phase in a quasicrystal (Benderski 1988). An earlier work also points out the part of defects: modulated phases involving defect networks were identified as precursor of the rhombohedral approximant of the $\mathrm{Al}-\mathrm{Cu}-\mathrm{Fe}$ quasicrystal (Audier 
et al 1991). The planar defects in the $\mathrm{Al}-\mathrm{Cu}-\mathrm{Fe}$ modulated phase are related to the facets of the approximant rhombohedral phase. A theoretical model of displacive transformation built in the 6-dimension space and using the Cut-and-Project algorithm account fairly well for this precursor state (Duneau 1992). A model of transformation mediated by phason walls has been proposed in which the quasicrystal transforms in a microstructure involving two approximant phases (Mosseri 1993). Theoretical works are usually taking the quasicrystal as the starting point of the transformation. To our knowledge, only the model proposed by Kulkarni et al (1993) addresses the reverse transformation:from crystal to quasicrystal. The authors derived, using the Cut-andProject method, a sequence of approximant crystals of increasing size which contains antiphase boundaries. Certainly, this model agrees with some of our observations in $\mathrm{Al}-\mathrm{Li}-\mathrm{Cu}$ but there is even more agreement with the example reported in the next section.

In the context of the study of defects in icosahedral motif phases, our attention has been attracted by the microstructure of the $\chi$-phase which frequently form particles after annealing in steels. These particles exhibit a dense network of defects strongly recalling the $\mathrm{Al}_{5} \mathrm{Li}_{3} \mathrm{Cu}$ phase microstructure except that the defects are non laying in well-defined planes.

\section{Core structure of intersecting antiphase boundaries}

The $\chi$-phase precipitating in steels has a composition close to the registered $\mathrm{Fe}_{18} \mathrm{Cr}_{6} \mathrm{Mo}_{5}$ phase, isostructural to $\alpha$-manganese $(a=0.89 \mathrm{~nm}, I 43 \mathrm{~m}, 58$ atoms/cell) (Bradley and Thewlis 1927). After a short annealing $(\approx 300 \mathrm{~h})$ at $700^{\circ} \mathrm{C}, \chi$-phase particles are obtained. They always exhibit a large density of boundaries which are separating small domains (figure 4). The domain size ranges from 20 to $200 \mathrm{~nm}$. Dark-field images in multi-beam conditions show that they are translation and not inversion domains. The fault vectors are identified by the extinction method in two-beam condition as simple translation vectors: $1 / 4\langle 111\rangle$. As in the $\mathrm{Al}_{5} \mathrm{Li}_{3} \mathrm{Cu}$ phase, $1 / 4\langle 111\rangle$ fault vectors correspond to antiphase boundaries. The occurrence of these APBs has lead us to a new description of the $\chi$-phase in terms of a packing of two types of polyhedron: the Friauf (or Z16) polyhedron typical of the Frank-Kasper phases and a distorted Z16

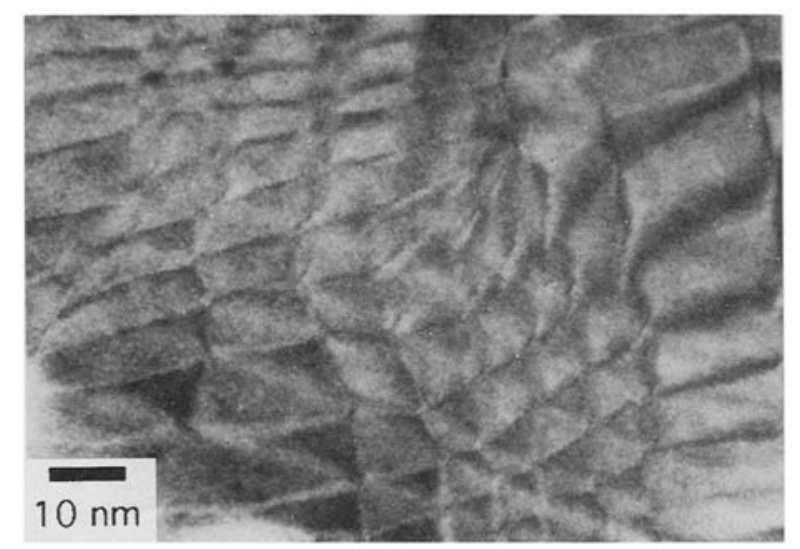

Figure 4. Crystalline $\chi$-phase particles exhibiting a network of APBs in $332 \mathrm{~h}$ annealed steels. 
polyhedron (Proult and Donnadieu 1997). The Friauf polyhedra are centred on the 000 and $1 / 21 / 21 / 2$ lattice nodes while the distorted Z16 polyhedra are centred on the $1 / 4$ $1 / 41 / 4,1 / 43 / 41 / 4,3 / 43 / 41 / 4$ and $1 / 43 / 43 / 4$ positions.

For long annealing time $(\approx 700 \mathrm{~h})$, the density of defects in the $\chi$-phase particles strongly increases (figure 5) and anomalous EDPs are obtained. Actually there is a contradiction: the EDPs taken in the selected area mode are aperiodic (figure 6) while the EDPs taken in the nanodiffraction mode are the ones of the $\chi$-phase. The existence of crystalline $\chi$-phase domains is also confirmed by the high resolution (HREM) images (figure 2). The domain size is $\sim 10 \mathrm{~nm}-20 \mathrm{~nm}$ and the crystalline domains are now separated by thick walls.

When EDPs are taken with a selected area aperture $(0.5 \mu \mathrm{m})$, they exhibit welldefined spots that no longer correspond to the $\chi$-phase crystal. Moreover, the EDPs are incompatible with a crystallographic organization. The global symmetry has been preserved: the EDPs are consistent with the $I 43 \mathrm{~m}$ space group but the diffraction spots are no longer periodically arranged (figure 6a).

The diffraction spots on the 3-fold and $\langle 011\rangle$ EDPs (figure 6b) also show an anomalous behaviour: the spots are split but the splitting depends from the spot position. No spot can be indexed as a $\chi$-phase one except the (033) spots. Only the mean positions of the split spots are at the $\chi$-phase spot positions. The deformation of the 4-fold EDP (figure 6a) is the most remarkable. As previously, the $\chi$-phase are absent except the 033 spots (labelled E). Long exposure times allow to see weak spots (labelled B and C) shifted from the $\chi$-phase spot positions. The ratios of the position relating the spots A, B, C, D along the $\langle 011\rangle$ direction have values close to $\tau$ (Golden ratio), $\tau^{2}$ and to $\sqrt{5}$.

The anomalous EDPs recorded in the long annealed samples recall a quasicrystalline phase observed in several rapidly solidified intermetallics: $\mathrm{V}-\mathrm{Ni}-\mathrm{Si}, \mathrm{Fe}-\mathrm{Cr}-\mathrm{Mo}$, the so-called modulated phases (Wither et al 1990; Carron and Portier 1994). The main difference with our observations is a much larger spot splitting in the rapidly solidified phases. The annealed microstructure appears as a precursor state of the modulated phase. The existence of crystalline domains and the identification of APB suggests that the anomalous microstructure involves two phases: the $\chi$-phase forming the small domains and another phase located either at the APB or in the APB intersection. In this

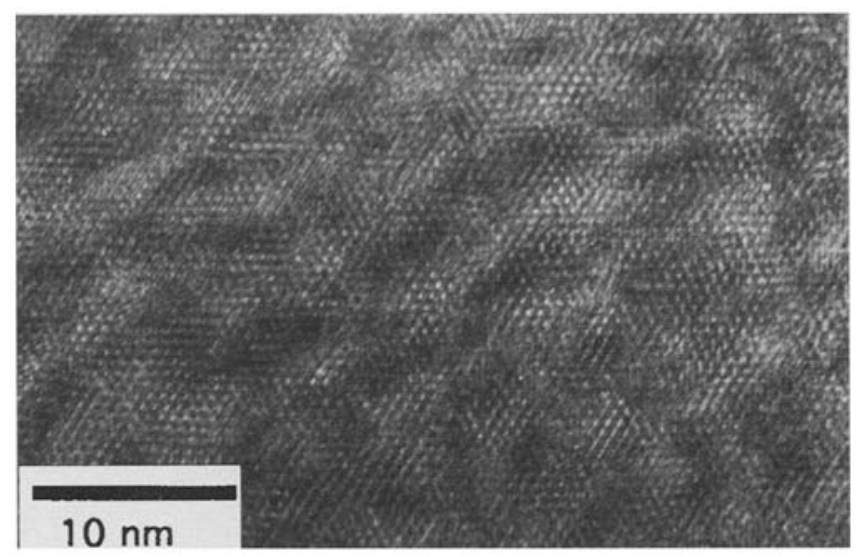

Figure 5. High resolution images of $\chi$-phase particles after a $664 \mathrm{~h}$ annealing. Note the width of the boundaries separating the crystalline domains. 

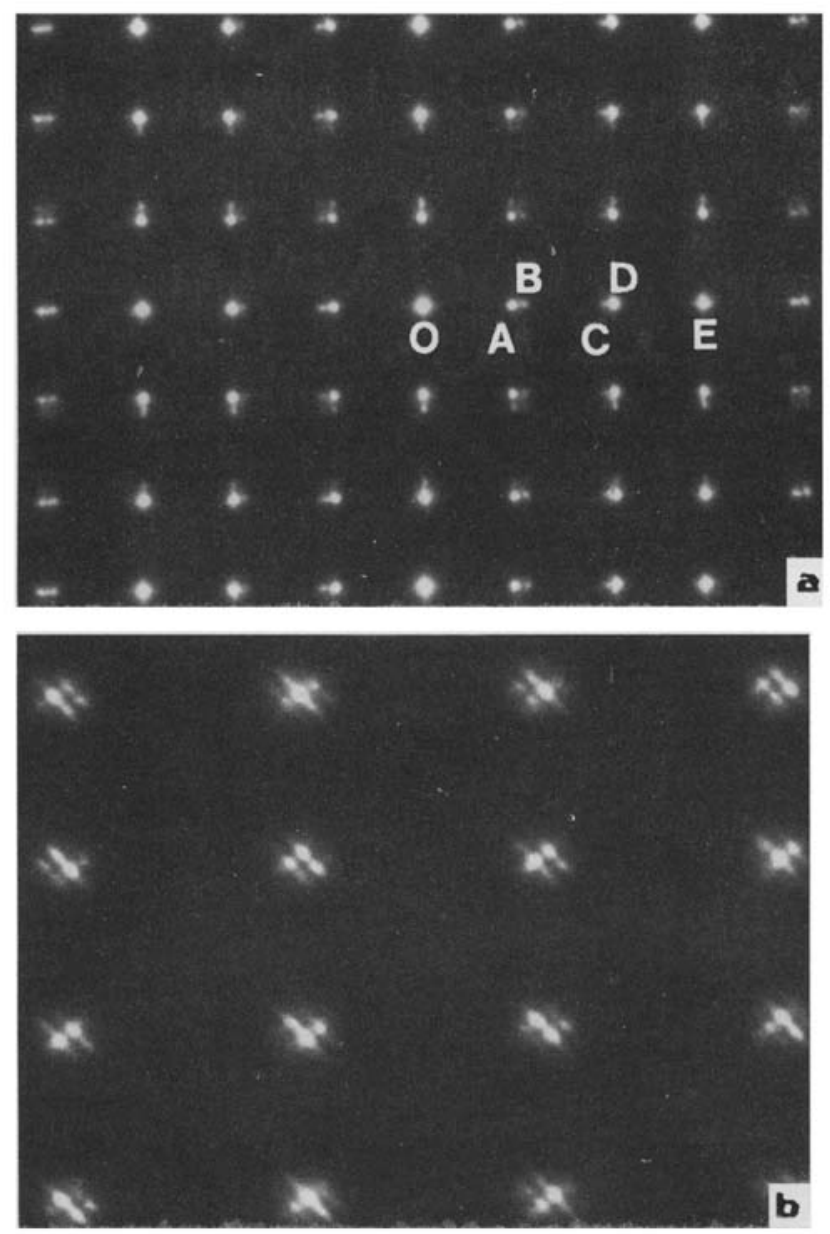

Figure 6. a. 4-fold aperiodic EDP from a $664 \mathrm{~h}$ annealed $\chi$-phase particle (figure 5). The spots labelled $A, B, C, D, E$ are related by particular ratio: $O C / O B=1 \cdot 6, O E / O B=2 \cdot 6$, $\mathrm{OD} / \mathrm{OA}=2.2$ and $\mathbf{b}$. $\langle 011\rangle$ zone axis EDP in an anomalous $\chi$-phase particle (figure 5). Note the particular splitting of the spots. Figures $6 a$ and $b$ are not at the same scale.

interpretation, the extinction of the $\chi$-phase spots simply results from small angular deviation between the crystalline domains. Such deviations are observed on the HREM images examined at glancing angle.

The hypothesis of two phases is usually tested with dark-field (DF) images. Here, the diffraction spots are too close to perform directly DF images. Therefore, HREM images have been digitized and their fourier transforms have been computed. On the numerical fourier transform, we select a peak equivalent to the spot $A$ in figure $6 \mathrm{a}$ and then compute the inverse fourier transform. The size of the aperture (in the fourier space) is large enough to avoid artifact in the images obtained by inverse fourier transform. The same procedure has been carried out with another selected peak (spot B). In both cases, we obtained an image with bright areas (not located in the same places). These numerical dark-field images confirm the hypothesis of a two-phase microstructure. 
Because of the remarkable ratio between the spot positions $\left(\tau, \tau^{2}\right.$ and to $\left.\sqrt{5}\right)$, we have proposed a preliminary interpretation in which the second phase is an icosahedral quasicrystal similar to the one observed in steels (Sidhom and Portier 1983). It is difficult to ascertain whether an icosahedral phase or rather a large parameter cr ystalline phase with icosahedral motifs is involved. However, the main point here is the existence of a coherent microstructure which generates well-defined spots in spite of the small domain sizes. This property suggests to examine in detail the core of intersecting boundaries.

A nice geometrical feature of $1 / 4\langle 111\rangle$ fault vectors consists in their combination without residual fault: $1 / 4[111]+1 / 4[-11-1]+1 / 4[1-1-1]+1 / 4[-1-11]=0$. Our new description of the $\chi$-phase structure (Proult and Donnadieu 1997) allows to build easily the vertex of four intersecting APBs. The core consists in the intersection of four distorted Z16 polyhedra which leads to a small tetrahedron contained in a larger tetrahedral shell. This double tetrahedron aggregate is never met in Frank-Kasper phase or phase with icosahedral motifs but is a basic cluster in another complex structure-type: the $\gamma$-brass structure $(a=0.89 \mathrm{~nm}, I 43 \mathrm{~m}, 52$ atoms/cell). A well-known member of the $\gamma$-brass structure-type is the $\mathrm{Cu}_{5} \mathrm{Zn}_{8}$ phase, an archetype of Hume-Rothery compounds (von Heidenstam et al 1968).

This result meets one of main features of the model proposed by Kulkarni et al (1993). In their model, the defect core of intersecting APBs is perfect and involves motifs which were not contained in the approximant phase. This is a key point in the transformation from crystal to quasicrystal since quasicrystal requires two types of tiles. Kulkarni et al (1993) modelized the phase transformation for 2-dimensional quasicrystals having 8-fold and 12-fold symmetry. Our observations agree with the main points of their model: APBs, continuous transformation, coherency of the microstructure due to perfect reconstruction and the generation of a new type of tiles at the intersection defect cores.

\section{Conclusion}

It is first remarkable that defects in the complex phases related to quasicrystals are simply antiphase boundaries. This property observed for two quite different systems ( $\mathrm{Al}-\mathrm{Li}-\mathrm{Cu}$ and $\mathrm{Fe}-\mathrm{Cr}-\mathrm{Mo}$ ) holds for the $\mathrm{Al}_{6} \mathrm{CuMg}_{4}$, isostructure of $\mathrm{Al}_{5} \mathrm{Li}_{3} \mathrm{Cu}$. In the two examples reported here, the density of defects is very high, meaning that APBs in both cases are low energy defects. In the first example (AlLiCu), the high density of defect network becomes first organized periodically and then aperiodically. The interaction between defects here is certainly due to long range electronic forces related to the Hume-Rothery nature of the $\mathrm{Al}_{6} \mathrm{Li}_{3} \mathrm{Cu}$ quasicrystal (Wither et al 1990; Carron and Portier 1989). On the contrary, in the $\mathrm{Fe}-\mathrm{Cr}-\mathrm{Mo}$ system, the defects even in high density do not become organized, still an anomalous behaviour is identified. It seems to result from the presence of a second phase at the APBs and the APB intersections. TEM investigation and reconstruction of the defect core support this interpretation. These observations meet the prediction of a model proposed for a continuous crystal/quasicrystal transformation. Local structures are then prominent features for the crystal/quasicrystal transformation in the $\mathrm{Fe}-\mathrm{Cr}-\mathrm{Mo}$ system. This behaviour is probably due to the presence of transition metals. Thus the present examples remind of two classical cases: the formation of long period structures by APB organization and 
the wetting of APB by another phase. Regarding the quasicrystalline state, the observations also suggest that geometrical frustration is responsible for the approximant/quasicrystal transformation.

\section{References}

Audier M, Bréchet Y, De Boissieu M, Guyot P, Janot Ch and Dubois J M 1991 Philos. Mag. 631375

Benderski L 1988 Mater. Sc. Ing. 99331

Blandin A, Friedel J and Saada G 1966 J. Phys. 7-8 C3-128

Bradley A J and Thewlis J 1927 Proc. R. Soc. A115 456

Carron D and Portier R 1994 Electron microscopy, in Application in material sciences, ICEM 13 (Paris: Les Editions de Physique) pp 667-668

Degand C, Wang K and Garoche P 1993 J. Non-Cryst. Solids 153-154 478

Donnadieu P 1991 Philos. Mag. 6497

Donnadieu P 1992 Philos. Mag. 6515

Donnadieu P 1994 J. Phys. I 4791

Donnadieu P and Degand C 1993 Philos. Mag. 68317

Duneau M 1992 J. Phys. I 21731

Frank F C and Kasper J S 1958 Acta Cryst. 11184

von Heidenstam O, Johansson A and Westman S 1968 Acta Chem. Scand. 22653

Hippert F and Gratias D (eds) 1994 Lectures on quasicrystals (Paris: Les Editions de Physique)

Janot Ch 1992 Quasicrystals, a primer, in Monographs on the physics and chemistry of materials (Oxford: Oxford Science Publications) Vol. 48

Kulkarni U D, Banerjee S and Kulkarni S D 1993 Acta Metall. 411283

Mosseri R 1993 J. Non-Cryst. Solids 153-154 658

Proult A and Donnadieu P 1995 Philos. Mag. 72337

Sainfort P and Dubost B 1986 J. Phys. 47321

Sidhom H and Portier R 1989 Philos. Mag. Lett. 59131

Wither R L, Feng Y C and Lu G H 1990 J. Phys.: Condens. Matter 23187 